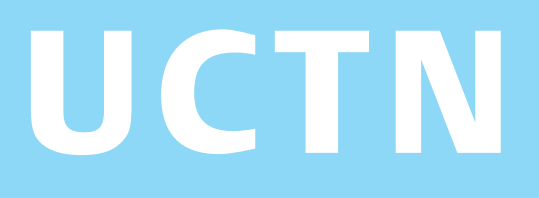

\title{
Capsule endoscopy in a child with a jejunal hemangioma
}

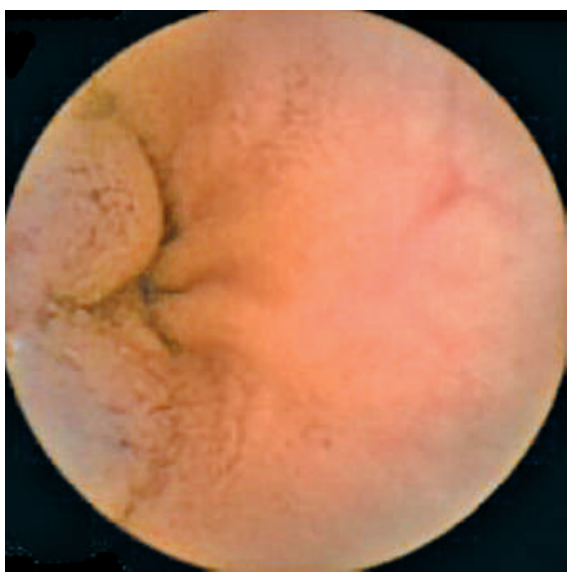

Figure 1 Capsule-endoscopic image of a purplish-blue lesion with a central depression, 196 min after passage of the pylorus. The lesion was suspected to be a hemangioma.

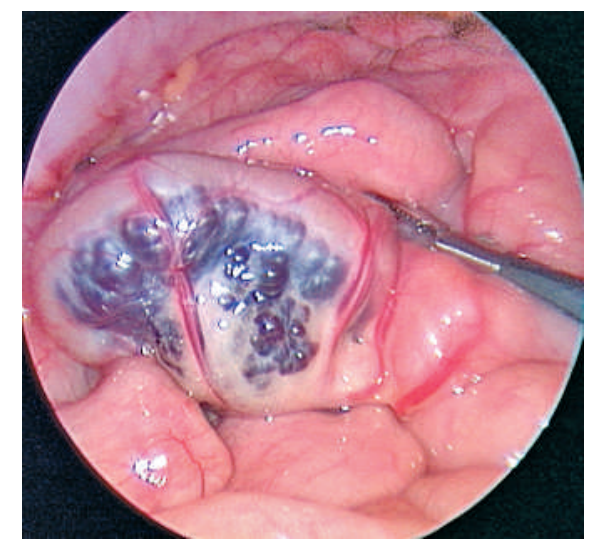

Figure 2 Laparoscopic image of the hemangioma.

A boy aged 2 years and 5 months was referred to our tertiary medical center due to recurrent melena, with a maximum fall in hemoglobin to $9.6 \mathrm{~g} / \mathrm{dl}$. Gastroduodenoscopy and ileocolonoscopy failed to detect a bleeding source. Although a Meckel's scan was negative, laparoscopy was carried out, as there was a strong clinical suspicion of a Meckel's diverticulum. No abnormalities were seen, and the boy was admitted to hospital to undergo

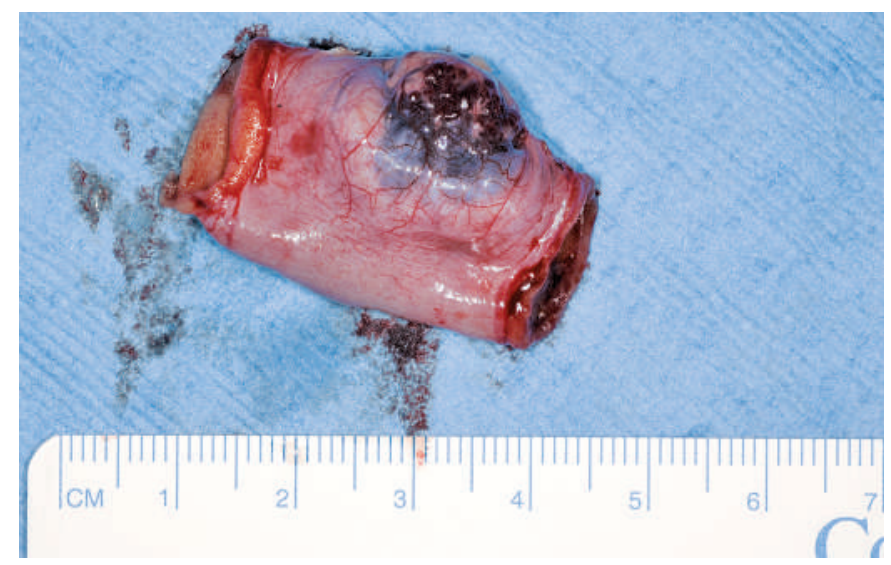

Figure 3 The surgical specimen with the jejunal hemangioma.

video capsule endoscopy. The capsule was introduced endoscopically with the patient under general anesthesia and passed uneventfully with the feces within $24 \mathrm{~h}$. On CE, a purplish-blue lesion with a central depression was seen 196 min after passage of the pylorus (Figure $\mathbf{1}$ ). The lesion was suspected to be a hemangioma. Laparoscopy was therefore carried out, demonstrating a hemangioma $2.2 \mathrm{~cm}$ long (Figure 2 ), which was subsequently resected (Figure $\mathbf{3}$ ).

Five months after surgical resection of the hemangioma in the jejunum, the boy was doing well and no further gastrointestinal bleeding had occurred. Capsule endoscopy was a useful diagnostic tool in this child with rectal blood loss and negative regular endoscopic and radiographic examinations.

Endoscopy_UCTN_Code_CCL_1AC_2AB Endoscopy_UCTN_Code_CCL_1AC_2AC

M. M. Tabbers ${ }^{1}$, K. F. Bruin ${ }^{2}$, M. A. Benninga ${ }^{1}$, D. Vieira-Travassos ${ }^{3}$, J. H. Oudshoorn ${ }^{4}$
${ }^{1}$ Dept. of Pediatric Gastroenterology and Nutrition/Emma Children's Hospital, Academic Medical Center, University of Amsterdam, Amsterdam, The Netherlands

2 Dept. of Gastroenterology and Hepatology, Academic Medical Center, University of Amsterdam, Amsterdam, The Netherlands

${ }^{3}$ Dept. of Pediatric Surgery/Wilhelmina Children's Hospital, University Medical Center, Utrecht, The Netherlands

${ }^{4}$ Dept. of Pediatric Gastroenterology and Nutrition/Wilhelmina Children's Hospital, University Medical Center, Utrecht, The Netherlands.

\section{Corresponding Author}

\section{M. Tabbers, M.D.}

Emma Children's Hospital, g8-260

Academic Medical Center

PO Box 22700

1100 DD Amsterdam

The Netherlands

Fax: $\quad+31-20-6917735$

E-mail: m.m.tabbers@amc.nl 